\title{
Secured Data Transmission in VANET Using Vehicular Digital Hash Gen Model
}

\author{
Rajasekar Rangasamy, H. Shaheen, T. Sreenivasulu, Nartkannai. K
}

\begin{abstract}
Vehicular adhoc structures (VANETs) handle the Public Key Infrastructure (PKI) and Certificate Revocation Lists (CRLs) for their security. In any PKI structure, the check of a got message is performed by checking if the check of the sender is joined into the current CRL, Verifying the reliability of the certification and standard for the sender. In this paper, it has been propose a Vehicular Digital Hash Gen show up (VDHG) for VANETs, which replaces the dull CRL checking process by a profitable revoking checking process. The renouncing check process in VDHG uses a Private Key Infrastructure (PKI), where the key used in finding the VDHG is shared particularly between On-Board Units (OBUs). In like manner, VDHG uses a novel probabilistic key stream, which extras with $O B U$ s to trade and revive an issue key. VDHG can on a very basic level lessen the data torment in light of the message declaration deferral pulled back and the standard assistance structures using CRL.
\end{abstract}

Keywords: VANETs, Vehicular Digital Hash Gen model, Time-Consuming, Private key infrastructure, message Verification.

\section{INTRODUCTION}

\section{Prologue to VANET}

Vehicular adhoc frameworks (VANETs) are a subgroup of adaptable adhoc structures (MANETs) with the particular property that within centers are vehicles like automobiles, trucks, transports and cruisers. This gathers center point improvement is obliged by parts like road course, consolidating traffic and traffic headings. In perspective on the obliged center point movement it is a reachable supposition that the VANET will be kept up by some settled structure that assists with a few affiliations and can offer access to stationary frameworks. The settled establishment will be passed on at central zones like slip ways, advantage stations, unsafe intermingling focuses or puts obviously refreshing for risky cools.

Focuses are required to pass on by procedures for North American DSRC standard that uses the IEEE 802.11p standard for remote correspondence. To permit correspondence with people out of radio range, messages must be sent by different focuses (multi-jump correspondence). Vehicles are not open to the serious vitality, space and figuring limits obstacles ordinarily got a handle on for MANETs. Additional testing is the possibly fast of the inside focuses (up to $250 \mathrm{~km} / \mathrm{h}$ ) and the extensive.

Revised Manuscript Received on August 14, 2019.

Rajasekar Rangasamy, Professor/Dept. of CSE, St. Peters Engineering College, Hyderabad, Telangana, India.

H.Shaheen, Professor/Dept. of CSE, St. Peters Engineering College, Hyderabad, Telangana, India..

T.Sreenivasulu, Professor/Dept. of CSE, St. Peters Engineering College, Hyderabad, Telangana, India.

Nartkannai.K, Professor/Dept. of CSE, St. Peters Engineering College, Hyderabad, Telangana, India.
An essential's VANET will probably build street security. To accomplish this, the vehicles go about as sensors and trade alerts or - all the more for the most part - telematics data (like flow speed, area or ESP action) that empowers the drivers to respond right on time to strange and possibly unsafe circumstances like mishaps, roads turned parking lots or coating. The data given by different vehicles and stationary framework may likewise be utilized for driver right hand frameworks like versatile journey control (ACC) or breaking partners. Furthermore, approved substances like police or fire-contenders ought to have the capacity to send caution signs and guidelines for example to clear their direction or stop other street clients. Other than that, the VANET should build comfort by methods for esteem included administrations like area put together administrations or Internet with respect to the street.

The ongoing appropriation of the different 802.11 remote benchmarks has caused an emotional increment in the quantity of remote information systems. Today, remote LANs are very sent and the expense for remote hardware is proceeding to drop in cost. As of now, a 802.11 connector or passage (AP) can be bought for by nothing. Because of the high acknowledgment of the 802.11 guidelines, the scholarly community and the business division are searching for other appropriate answers for these remote innovations. Versatile specially appointed systems (MANET) are one territory that has as of late gotten impressive consideration. One promising utilization of versatile impromptu systems is the improvement of vehicular specially appointed systems trim structure, work unified. inside a strikingly named framework goes about switch. obsessions framework by remote visit various center obsessions in their sensibly upside arbitrary frameworks is it is possible to pass on structures in zones where it isn't sound demonstrate required establishment. over the top ridiculous adjust 802.11 districts with spread a colossal bit of the courses in the United States. Another favored position of without any planning structures immediately passed on official affiliation. relationship of a basic scale vehicular structure would be a cumbersome endeavor. These reasons add to the unrehearsed structures being connected with vehicular conditions. Traffic fatalities are one of the standard wellsprings of death in the United States. The Federal Communications Commission (FCC), understanding the issue of traffic fatalities in the US submitted $75 \mathrm{MHz}$ of the broken interface in the range 5.850 to $5.925 \mathrm{GHz}$ to be used for vehicle to vehicle and vehicle to roadside correspondence. The $5.9 \mathrm{GHz}$ range was named Dedicated 
Short Range Communication (DSRC) and relies upon a saving of 802.11a. Seven channels of $10 \mathrm{MHz}$ each make up DSRC, with six of the channels being used for affiliations and one channel for control. point of convergence of the undertaking is to draw in the driver of a vehicle to get data about their mixing condition. The control channel is utilized to pass on movement messages for example to alert the driver of conceivably perilous street conditions. The control incorporate is in like way used to report the affiliations that are open. In the event that vehicle finds a relationship of imperativeness on the control channel, it, changes to one of the alliance channels to utilize the association. A bit of extra respect added highlights are to be given by the association channels, for example, the affirmation of spots of significance for the driver's area for example bistros in the domain or gas costs.

\section{RELATED WORKS}

These making headway exchanges (VC) raises diverse explicit issues that should be tended to affirmation head for . In this position paper, we are worried over security and character the board regarding these structures. We see VCexpress issues and inconveniences, thinking about the striking highlights of these structures. Specifically, we see them as for other continuously wide security affirmation endeavors, correspondingly association, adaptable remote correspondence types of progress.

\section{Train: Providing Location Privacy for VANET}

In vehicular adhoc structures (VANET), it is conceivable to find and pursue a vehicle subject to its transmissions, amidst correspondence with different vehicles or the street side framework. This sort of following prompts dangers on the region protection of the vehicle's client. Giving area protection VANET by enabling vehicles to counteract following of their communicate correspondences. We first, distinguish the one of a kind attributes of VANET that must be viewed as when planning appropriate area security arrangements. In light of these perceptions, it has been propose an area protection plot called CARAVAN, and assess the security upgrade accomplished under some current standard limitations of VANET applications, and within the sight of a worldwide enemy.

\section{DCS: An Efficient Distributed Certificate Service Scheme} for Vehicular Networks

It has been profitable dispersed attestation advantage (DCS) plot structures. Strategy adaptable assistance advantage in aces a noteworthy course exhibited revive insistence open structure (RSUs) in a precious way. In like manner, the DCS plan exhibits a by and large group check procedure for bearing witness to certification based inscriptions, which on a key level abatements the confirmation overhead. Security appraisal and execution assessment display that the DCS plan can reduce the motivation of assertion the board and achieve grand security and profit for vehicular correspondences.

\section{Verifies Vehicular Ad Hoc Networks}

Extraordinarily picked structures are another remote frameworks affiliation perspective for adaptable hosts. It has been formed a sharp vehicle structure. The ITS (cautious vehicle system) hardens two significant limit modules: Information getting ready application structure and Road condition information trading system. The key endeavor of the road condition information moving module is accountable for the information exchange of the vehicle inside, The module works unconstrained framework, we call the structure VANET (vehicular phenomenally picked framework). Vehicular structures are likely going to wrap up the most relevant sort of flexible unrehearsed frameworks. For guaranteeing the structure can run customarily, the information can be trading completely and fleetly, the security of VANET (vehicular uncommonly picked framework) of the road condition information trading system is fundamental. So join the characteristics of adhoc sort out itself, in the ITS of this paper, we concern the security issues of VANETs from a few of view and give up the reasonable lighting measures. To guarantee the ITS can be used under the security plan.

It proposes a productive pseudonymous verification plot with solid security protection (PASS), for vehicular correspondences. Not at all like conventional pseudonymous verification conspires, the span of the endorsement disavowal direct quantity denied and inconsequential what number of testaments repudiated underpins helped appropriated declaration benefit that enables the vehicles to refresh endorsements on street, yet the administration overhead is practically disconnected to the quantity of refreshed testaments. Moreover, PASS gives solid protection safeguarding to the vehicles with the goal that the foes can't follow any vehicle, despite the fact that the sum total of what RSUs have been imperiled. Broad reproductions exhibit that PASS outflanks recently detailed plans as far as the repudiation cost and the testament refreshing overhead.

\section{RESULTS \& DISCUSSIONS}

\section{A. Vehicle-to-Vehicle (V2V)}

In this Module, the two head correspondence modes, which self-rulingly award OBUs to talk with each other and with the foundation RSUs. Since vehicles give through remote channels, a strategy of ambushes, for example, blending false data, changing and replaying the spread messages can be activated.

A security strike on VANETs can have shocking unsafe or savage results to affirmed clients. Appropriately, guaranteeing secure vehicular correspondences is a sure need before any VANET application can be joined. A general saw answer for secure VANETs is to send Public Key Infrastructure (PKI), and to utilize Certificate Revocation Lists (CRLs) for dealing with the denied certificate. In PKI, each zone in the structure holds a real certificate, and each message ought to be intentionally restricted before its transmission. A CRL, for the most part issued by a Trusted Authority (TA), is a structure containing all the denied certificate. In a PKI structure, the help of any 
message is performed by first checking if the sender's certificate is joined into the current CRL, i.e., checking its denying status, by at that point, displaying the veracity of the sender' certificate, _and finally checking the sender's imperfection on the got message.

\section{B. Vehicular Digital Hash Gen (VDHG) structure}

a. Trusted Authority (TA): This is responsible for giving up checked certificate and Distributing puzzler keys to all OBUs in the structure.

b. Roadside units (RSUs): which are fixed units scattered wherever all through the structure. The RSUs can examine securely with the TA.

c. On-Board Units (OBUs): which are displayed in vehicles? OBUs can discuss either with various OBUs through $\mathrm{V} 2 \mathrm{~V}$ correspondences or with RSUs through V2I trades.

It will make Mac address and Digital Signature for data transmission.

\section{Security Analysis:}

\section{a. Hash Chain Values}

The estimations of the hash chains are always used in the renouncing structures, and thusly, the TA can eat up all the hash chain regards. As such, there should be a piece to

\section{b. Resistance of get-together strikes}

To pass on the denial check of any prepared unit an attacker needs to find the present issue. Moreover, find the TA befuddle key and scratching. To the denying check and TA message and drawing are unforgeable.

\section{c. Forward stupor}

The estimations of the hash chain joined into the renouncing messages are released to non-denied OBUs starting from the last estimation of the hash chain, and given the way where that a hash work is irreversible, a disavowed OBU can't use a hash chain regard got in a past renouncement structure to get the present hash chain regard, a denied OBU can't strengthen its business key set.

\section{d. Resistance to replay strikes}

Each message of an OBU joins directly at this very moment stamp in the repudiating check regard check an assailant can't record REV check at time $\mathrm{T}$ and replay it at last inside the not all that removed future structure as the tolerant OBU looks.below the figures; table heads should appear above the tables. Insert figures and tables after they are cited in the text. Use the abbreviation "Fig. 1", even at the beginning of a sentence.

\section{METHODOLOGY}

\section{A. Vehicular Digital Hash Gen (VDHG) procedure}

In VDGH procedure the flag that should be sent will be anchored by giving Hash an incentive to the clients. It additionally keeps up the computerized mark with the goal that a client will be spoken to extraordinarily through it. it additionally Hashing strategy a useful renouncing methodology utilizing smart and This reasonable for VANETs similarly with respect to any system utilizing a unstick the present hash chain with another.

PKI structure. To the best of our insight, this is the basic reaction for diminish the insistence concede happening because of checking the validness in VANETs.

\section{B. Private Key Infrastructure (PKI)}

An open key framework (PKI) is a lot of jobs, approaches, and systems expected to make, oversee, convey, use, store, and disavow advanced certificates[1] and oversee open key encryption. The reason for a PKI is to encourage the safe electronic exchange of data for a scope of system exercises, for example, web based business, web managing an account and secret email. It is required for exercises where straightforward passwords are a lacking verification strategy and increasingly thorough evidence is required to affirm the personality of the gatherings associated with the correspondence and to approve the data being transferred.[2]

In cryptography, a PKI is a game plan that ties open keys with individual personalities of elements (like people and associations). The coupling is built up through a procedure of enlistment and issuance of authentications at and by an endorsement expert (CA). Contingent upon the affirmation dimension of the authoritative, this might be done by a robotized procedure or under human supervision.

The PKI job that guarantees substantial and right enrollment is called enlistment specialist (RA). A RA is in charge of tolerating demands for computerized testaments and confirming the element making the demand. In a PKI, an enrollment expert is generally called a subordinate CA.

\section{Attestation Revocation Lists (CRLs)}

A validation denied instance, found statement master inappropriately help, endangered. Supports besides prevented dissatisfaction from securing clear substance hold keen method fundamentals, for example, spread of false reports, mis-delineation of programming conduct, or infringement of some other perspective appeared by the CA regulator or its client. The most comprehensively watched explanation behind repudiation is the client never again being in sole commitment concerning This reversible status can be utilized to watch the vaporous nonappearance of the check (e.g., client is faulty event that, in this model, the private key was found and no one advanced toward it, the status could be reestablished, and the request is liberal once more, in that point of confinement ousting the supporting from future CRLs.

\section{CONCLUSION}

We have proposed VDHG for VANETs, which engages message support by superseding the horrid CRL checking process with a snappy denial checking procedure using PKI work. The proposed VDHG uses a novel key sharing area which draws in an OBU to invigorate its exchanged off keys paying little character to whether it beginning late missed some refusal messages. In like manner, VDHG has an isolated part rendering it integrable with any PKI structure. Furthermore, it is safe to standard strikes while defeating the confirmation system using the standard CRL. In this way, 
VDHG can in a general sense decline the message trouble degree as a result of message statement deferral meandered from the standard certification structures using CRL checking. Our future work will focus on the assistance and message imprint check stimulating.

\section{REFERENCES}

1. P. Papadimitratos, A. Kung, J.P. Hubaux, and F. Kargl, "Privacy and Identity Management for Vehicular Communication Systems: A Position Paper," Proc. Workshop Standards for Privacy in User- Centric Identity Management, July 2006.

2. K. Sampigethaya, L. Huang, M. Li, R. Poovendran, K. Matsuura, and K. Sezaki, "CARAVAN: Providing Location Privacy for VANET," Proc. Embedded Security in Cars (ESCAR) Conf., Nov. 2005.

3. A. Wasef, Y. Jiang, and X. Shen, "DCS: An Efficient Distributed Certificate Service Scheme for Vehicular Networks," IEEE Trans. Vehicular Technology, vol. 59, no. 2 pp. 533-549, Feb. 2010.

4. M. Raya and J.-P. Hubaux, "Securing Vehicular Ad Hoc Networks," J. Computer Security, vol. 15, no. 1, pp. 3968, 2007.

5. Y. Sun, R. Lu, X. Lin, X. Shen, and J. Su, “An Efficient Pseudonymous Authentication Scheme with Strong Privacy Preservation for Vehicular Communications," IEEE Trans. Vehicular Technology, vol. 59, no. 7, pp. 3589-3603, Sept. 2010.

6. R. Lu, X. Lin, H. Luan, X. Liang, and X. Shen, "Pseudonym Changing at Social Spots: An Effective Strategy for Location Privacy in Vanets," IEEE Trans. Vehicular Technology, vol. 61, no. 1, pp. 86-96, Jan. 2012.

7. US Bureau of Transit Statistics, http://en.wikipedia.org/wiki/Passenger_vehicles_in_the_ United_States, 2012.

8. J.J. Haas, Y. Hu, and K.P. Laberteaux, "Design and Analysis of a Lightweight Certificate Revocation Mechanism for VANET," Proc. Sixth ACM Int'l Workshop VehiculAr InterNETworking, pp. 89-98, 2009.

9. IEEE Std 1609.2-2006, IEEE Trial-Use Standard for Wireless Access in Vehicular Environments - Security Services for Applications and Management Messages, IEEE, 2006.

10. "5.9 GHz DSRC," http://grouper.ieee.org/groups/scc32/dsrc/ index.html, 2012.

11. A. Wasef and X. Shen, "MAAC: Message Authentication Acceleration Protocol for Vehicular Ad Hoc Networks," Proc. IEEE GlobeCom, 2009.

12. J.P. Hubaux, "The Security and Privacy of Smart Vehicles," IEEE Security and Privacy, vol. 2, no. 3, pp. 49-55, May/June 2004.

13. A. Studer, E. Shi, F. Bai, and A. Perrig, "TACKing Together Efficient Authentication, Revocation, and Privacy in VANETs," Proc. IEEE CS Sixth Ann. Conf. Sensor, Mesh and Ad Hoc Comm. And Networks (SECON '09), pp. 1-9, 2009.

14. M. Raya, P. Papadimitratos, I. Aad, D. Jungels, and J.-P. Hubaux, "Eviction of Misbehaving and Faulty Nodes in Vehicular Networks," IEEE J. Selected Areas in Comm., vol. 25, no. 8, pp. 1557- 1568, Oct. 2007.

15. P.P. Papadimitratos, G. Mezzour, and J. Hubaux, "Certificate Revocation List Distribution in Vehicular Communication Systems," Proc. Fifth ACM Int'l Workshop VehiculAr Inter-NETworking, pp. 86-87, 2008.

16. Dr.T.Sreenivasulu, Dr.H. Shaheen, Dr.Rajasekar Rangasamy, "Web Innovation with IOT in Social
Environment for Sharing Efficient Information ", "International Journal of Mechanical and Production Engineering Research and Development (IJMPERD)",ISSN (P): 2249-6890; ISSN (E): 2249-8001 Vol. 8, Special Issue 6, Aug 2018, 717-729

17. Dr.Rajasekar Rangasamy, Dr.H. Shaheen, "Secured Message Exchange in Mission Critical Infrastructure using Conditional Privacy Preserving Authentication", "International Journal of Computer and Mathematical Science", Volume 7, Issue 5, May 2018, ISSN 2347 8527.

\section{AUTHORS PROFILE}

Rajasekar Rangasamy Professor/Dept. of CSE, St. Peters Engineering College, Hyderabad.

H.Shaheen Professor/Dept. of CSE, St. Peters Engineering College, Hyderabad.

T.Sreenivasulu Professor/Dept. of CSE, St. Peters Engineering College, Hyderabad.

Nartkannai.K Professor/Dept. of CSE, St. Peters Engineering College, Hyderabad. 